\title{
Colistin resistance in Gram-negative bacteria analysed by five phenotypic assays and inference of the underlying genomic mechanisms
}

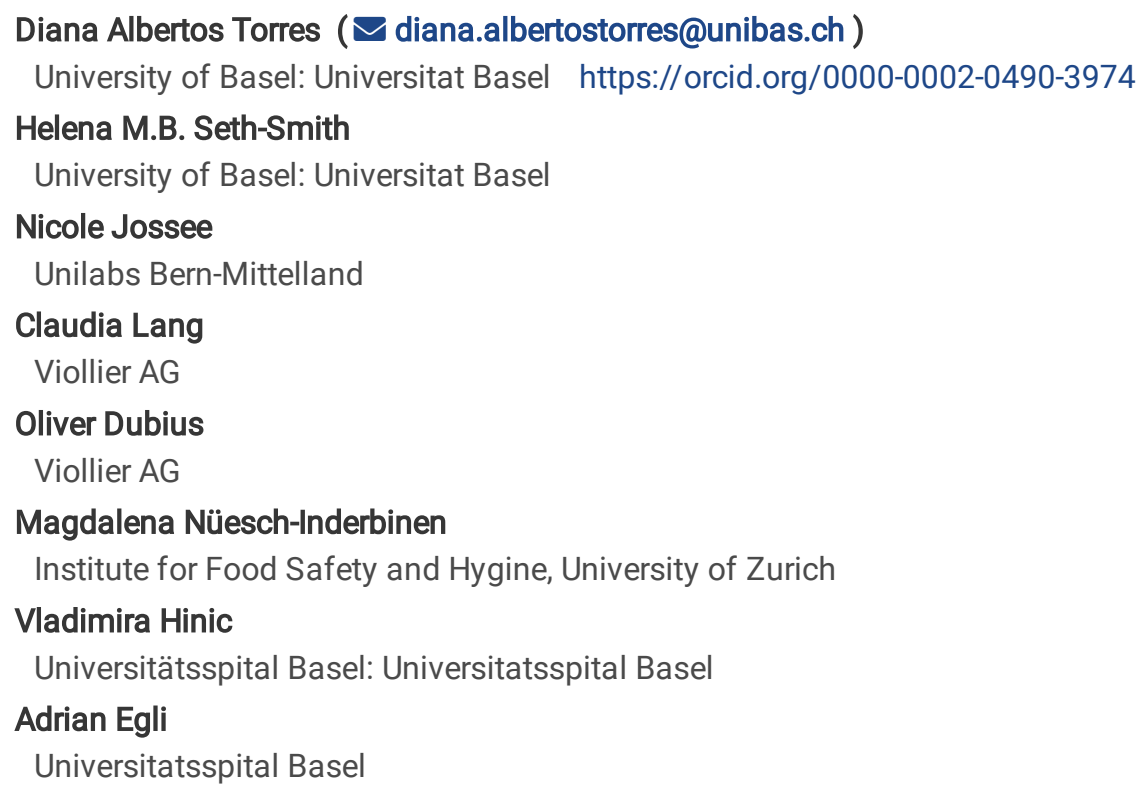

\section{Research article}

Keywords: colistin, resistance, antimicrobial susceptibility testing, whole genome sequencing, chromosomal mutations, plasmidmediated resistance

Posted Date: June 14th, 2021

DOI: https://doi.org/10.21203/rs.3.rs-610993/v1

License: (c) (1) This work is licensed under a Creative Commons Attribution 4.0 International License. Read Full License

Version of Record: A version of this preprint was published at BMC Microbiology on November 20th, 2021. See the published version at https://doi.org/10.1186/s12866-021-02388-8. 


\section{Abstract \\ Background}

Colistin is used against multi-drug resistant pathogens, yet resistance emerges either through dissemination of plasmid-mediated genes $(m c r)$ or chromosomal mutation of genes involved in lipopolysaccharide synthesis (i.e. $m g r B, p h o P Q, p m r C A B$ ). Phenotypic susceptibility testing is challenging due to poor diffusion of colistin in agar media, leading to an underestimation of resistance. We aimed to compare the performance of four different phenotypic approaches in the context of different molecular mechanism of resistance.

\section{Methods}

We compared the performance of Vitek $2 \circledast$ (bioMérieux, AST N242), Colistin MIC Test Strip (Liofilchem Diagnostici), UMIC (Biocentric), and Rapid Polymyxin NP Test (ELITechGroup) against the standard broth microdilution (BDM) method. We used whole genome sequencing (WGS) to infer the molecular mechanisms of resistance. A total of 97 Enterobacterales and non-fermenting bacterial isolates were collected from clinical samples during 2016-2017 and tested for colistin susceptibility. Data was analysed by comparing the susceptibility category (susceptible or resistant) and minimal inhibitory concentrations (MIC). We determined diversity of isolates by core genome multi locus sequencing typing (cgMLST) and identified antimicrobial resistance genes using NCBI and CARD databases.

\section{Results}

Of the 97 clinical isolates, 54 (56\%) were resistant by the standard broth microdilution. The highest susceptibility category concordance was achieved by the Rapid Polymyxin NP test (98.8\%) followed by UMIC (97.9\%), E-test MIC strip (96.9\%) and Vitek 2 (95.6\%). The highest MIC concordance was achieved by UMIC (80.4\%), followed by Vitek 2 (72.5\%) and E-test MIC strip (62.9\%). Among the resistant isolates, $23 / 54$ (43\%) were intrinsically resistant to colistin, whereas $31 / 54$ (57\%) isolates had acquired colistin resistance. Of these, $m c r-$ 1 was detected in four isolates and $m c r-2$ in one isolate. Mutations in $m g r B, p h o Q, p m r A, p m r B$, and $p m r C$ genes that led to amino acid changes were encountered in Klebsiella pneumoniae, Escherichia coli, and Acinetobacter bereziniae resistant isolates.

\section{Conclusions}

The Rapid Polymyxin NP test showed the highest categorical concordance and the UMIC test provided MIC values with a high concordance to the standard method. We found colistin resistance in diverse species occurred predominantly through spontaneous chromosomal mutation rather than plasmid-mediated resistance.

\section{Introduction}

Colistin is an antimicrobial agent of the polymyxin class. Although still widely used in veterinary medicine, colistin usage in human medicine was initially restricted to topical administrations due to its nephrotoxic and neurotoxic properties if given systemically(1). However, due to the recent dissemination of multidrug resistant (MDR) bacteria around the world, colistin has been increasingly used as a last resort antimicrobial for treatment of difficult-to-treat infections caused by MDR Gram-negative pathogens(1-3).

Colistin is a cationic polypeptide containing an acylated tripeptide chain at its $\mathrm{N}$-terminus responsible for the toxicity of colistin. The mechanism of action relies on the interaction of the hydrophobic region of the fatty acid and phosphate groups of the lipid $A$ of the lipopolysaccharide (LPS). This interaction displaces the divalent cations that naturally stabilize the outer bacterial membranes leading to leakage of cellular compounds and, ultimately, cell death(4-6). Although this is the main mode of action, other mechanisms have been described such as inhibition of respiratory enzymes NDH-2 $(5,7,8)$ and neutralization of the LPS, which may help prevent septic shock(9). Due to its mechanism of action, colistin is highly effective against most Enterobacterales species and non-fermenting Gram-negative bacteria such as Acinetobacter baumannii and Pseudomonas aeruginosa. Conversely, colistin is not active against Gram-positive bacteria, Gram-negative cocci, and anaerobic bacteria. Some Enterobacterales species are intrinsically resistance to colistin, such as Serratia marcescens, Morganella morganii, and Proteus mirabilis due to the constitutive expression of genes (i.e. eptB) that lead to the modification of the LPS and its increase of charge $(4,5)$. Hafnia spp. have also been suggested to be intrinsically resistant(10). 
Bacteria have developed resistance mechanisms against colistin mainly through the modification of the LPS. This is achieved by the addition of 4-amino-4-deoxy-L-arabinose (L-Ara-4N) or phosphoethanolamine ( $\mathrm{pEtN})$ to lipid A that increases the positive charge of LPS and thus reduces its affinity to colistin $(5,6,11)$. The synthesis and addition of L-Ara- $4 \mathrm{~N}$ and pEtN is mediated by the PmrAB and $P$ hoPQ two-component system genes and its regulators genes (i.e. $\mathrm{mgrB}$ ) but also through plasmid-mediated genes like mobile colistin resistance $(m c r)(5,11-13)$. Colistin resistance by alteration of LPS has been widely described in several species like $K$. pneumoniae and E. coli(4,5,14-18). Other species, like $A$. baumannii acquire resistance due to the complete loss of LPS by inactivation of the lipid A biosynthesis genes $(I p x A, I p x C$ and $I p x D)(5,19)$.

In view of concerning emergence of resistance, it is of critical importance that reliable tools for susceptibility testing are available. However, susceptibility testing is a challenge due to the cationic nature of colistin, which causes it to adhere to the negatively charged polystyrene surfaces used in routine laboratory plates(20), but also due to its poor diffusion in agar because of its large molecular size. In 2016 EUCAST warned about the difficulties of colistin testing using disk diffusion and gradient test, as these methods seem to underestimate resistance. EUCAST recommends broth microdilution (BDM) as the only valid method and that it should be performed with sulphate salts of polymyxins in cation-adjusted Mueller-Hinton broth, without any additives like polysorbate-80 (P-80) in trays made of polystyrene(21). Nevertheless, this method may be difficult to implement in a routine diagnostic laboratory since other assays, such as disk diffusion or automated testing like Vitek ${ }^{\circledR}$ or BD Phoenix $\mathrm{x}^{\mathrm{TM}}$, are commonly used for colistin susceptibility testing and are often part of laboratory automation.

The goal of our study was to first compare the performance of four different diagnostic assays for colistin susceptibility testing: Vitek $2 \circledast$ (bioMérieux, AST N242), Colistin MIC Test Strip (Liofilchem Diagnostici), UMIC (Biocentric), and Rapid Polymyxin NP Test (ELITechGroup). We aimed to find the most accurate, robust and easy-to-perform assay suitable for the daily usage in the routine microbiology laboratory. Standard broth microdilution method (BDM) was used as reference standard. Our second aim was to determine the underlying genetic mechanisms of resistance using whole genome sequencing and compare this against the MICs.

\section{Materials And Methods}

\section{Ethical statement.}

All strains were collected as part of quality control purposes and establishment of new diagnostic assays. All strains were used in anonymized way and no clinical data was collected. For these quality control studies no ethical approval is necessary according to the Human Research Act in Switzerland.

\section{Clinical isolates and culture conditions.}

We used 97 isolates from the Enterobacterales order and non-fermenting bacteria from clinical samples in the period from 2016 to 2017 at the clinical microbiology laboratories of the University Hospital Basel, Switzerland; Cantonal Hospital Lucerne, Switzerland and laboratory Viollier in Allschwil, Switzerland (Additional file 1). All colistin resistant isolates tested with Vitek $2 \circledast$ (bioMérieux, Marcy'l Etoile, France) from the University Hospital Basel were included in the study. We used Escherichia coli NCTC-13846 and E. coli KP37 harbouring $m c r-1$ and $m c r-2$, respectively, as reference strains(22). The strain collection also included 43 colistin sensitive isolates.

Species identification was performed at the time of diagnosis with matrix-assisted laser desorption ionization time of flight mass spectrometry MALDI-TOF MS (MALDI-TOF MS; Bruker, Bremen, Germany) by using the mass-spectrum library and the MALDI Biotyper 3 software (OC 3.1, Bruker Daltonics) at standard conditions. All bacterial isolates were frozen at $-70^{\circ} \mathrm{C}$ in cryogenic Microbank ${ }^{\text {TM }}$ vials (ProLab Diagnostics, Birkenhead, UK). Prior to testing, the strains were cultured on Columbia agar supplemented with $5 \%$ sheep blood (BD Diagnostic Systems, Allschwil, Switzerland) with subsequent subculture after 24 hours.

Assays for colistin susceptibility testing.

Standard BDM was performed according to EUCAST recommendations by using 11 concentrations ranging from 0.06 to $64 \mathrm{mg} / \mathrm{mL}$ including a growth control without colistin. Colistin susceptibility testing with Vitek $2 \circledast$ (bioMérieux) was performed by using the AST $\mathrm{N} 242$ card. For calculation of colistin MIC, the following dilutions were tested: $4 \mathrm{mg} / \mathrm{ml}, 16 \mathrm{mg} / \mathrm{ml}$ and $32 \mathrm{mg} / \mathrm{ml}$. MIC determination was performed with Colistin MIC Test Strip (Liofilchem Diagnostici, Roseto degli Abruzzi, Te, Italy) (Additional file 2). UMIC is a manual broth microdilution test and was performed according to the manufacturer's instructions. Briefly, a 1:200 dilution of a 0.5 Mc Farland solution of bacteria in Mueller-Hinton II broth was inoculated in the UMIC strips and incubated in a humid atmosphere at $35-37^{\circ} \mathrm{C}$ for 18 hours. The MICs were read visually (turbid = growth, clear = no growth) (Additional file 2). Rapid Polymyxin NP Test (ELITechGroup) is based on 
the colourimetric detection of rapid glucose metabolism associated with bacterial growth, through a pH indicator colour change from orange to yellow, in the presence of a defined concentration of colistin. The test was read after two and three hours of incubation and the results were recorded as either colistin susceptible or colistin resistant (without MIC value) (Additional file 2). Reading of all assays was performed with two independent persons in a blinded fashion. If the results were discrepant, the testing was repeated. The MIC variation of \pm 1 titre compared to reference MIC was considered as concordant. All minimal inhibitory concentrations (MICs) were interpreted according to EUCAST Version 10.0, 2020(23).

Whole genome sequencing and antimicrobial resistance gene detection.

All isolates underwent whole genome sequencing on an Illumina Miseq 2x300bp or NextSeq 2x150bp after NexteraXT library preparation to mean coverage over $35 x$. Assembly was performed, after trimming with trimmomatic v 0.38(24), with Unicycler v0.3.0b(25) using standard settings. The assemblies were annotated with Prokka v1.13(26) and ABRicate v0.8.10(27) was used to search for species identification, antimicrobial resistance genes and phylogeny context. Species were identified using ribosomal MLST(28) and ANI comparisons for Klebsiella spp. Where discrepancies in species classification between the original MALDI-TOF MS identification and ribosomal MLST identification from whole genome sequencing data occurred, in particular, classification of isolates as Klebsiella oxytoca or Klebsiella michiganensis, and between species belonging to the E. cloacae complex, namely E. cloacae, E. homaechei and E. bugandensis, the latter was taken as accurate. We determined antimicrobial resistance genes using the NCBI or CARD AMR gene databases. Assemblies were typed by core genome multi-locus sequencing typing (cgMLST) within Ridom Seqphere+ (Münster, Germany) using relevant published schemes where available(29) or ad hoc schemes where unavailable for that species. Detailed searches of genome assemblies from resistant isolates and also from susceptible isolates as negative controls, were performed using visualization in Artemis v 18.1.0(30), with alignments in Jalview v 2.11.1.0(31).

\section{Results}

Phenotypic colistin resistance in the strain collection.

Of the isolates tested ( $n=97), 54(56 \%)$ were resistant to colistin by the standard broth microdilution testing and $43(44 \%)$ were susceptible. Among the resistant isolates, 23 (43\%) belonged to the bacterial species $P$. vulgaris, P. mirabilis, S. marcescens and $H$. alvei and thus possess intrinsic resistance. Whereas 31 (57\%) belonged to Acinetobacter spp., E. cloacae, E. aerogenes, E. coli, $K$. pneumoniae, K. oxytoca and $P$. aeruginosa which display various mechanisms of acquired resistance. Among these latter nonintrinsically resistant isolates, three $(10 \%)$ had a MIC value of $4 \mathrm{mg} / \mathrm{L}$, nine $(29.0 \%)$ had a MIC of $8 \mathrm{mg} / \mathrm{L}$, eight $(26 \%)$ had a MIC of $16 \mathrm{mg} / \mathrm{L}$, seven $(23 \%)$ had a MIC value of $32 \mathrm{mg} / \mathrm{L}$, and four (13\%) isolates displayed a MIC of $\geq 64 \mathrm{mg} / \mathrm{L}$ (Figure 1$)$. A total of $19(20 \%)$ isolates had a high MIC value, considered in this study as $\geq 16 \mathrm{mg} / \mathrm{ml}$, which belonged to the species Acinetobacter spp. $(\mathrm{n}=1), E$. aerogenes $(\mathrm{n}=1)$, E. cloacae $(\mathrm{n}=1)$, E. coli $(\mathrm{n}=4), K$. oxytoca $(\mathrm{n}=2)$ and K. pneumoniae $(\mathrm{n}=10)$.

Rapid polymyxin NP Test shows highest concordance with susceptibility category and UMIC with MIC measurements.

Susceptibility category concordance (susceptible or resistant) between the broth dilution gold standard and the test protocols (Vitek $2{ }^{\circledR}$, Colistin MIC Test Strip, UMIC and Rapid Polymyxin NP Test) is shown in Table 1. The highest overall concordance was achieved with Rapid Polymyxin NP test (98.8\%), followed by UMIC (97.9\%), colistin E-test MIC strip (96.9\%), and Vitek 2 (95.6\%). We calculated the sensitivity and specificity for the susceptibility category according to the BDM reference standard in all isolates. The highest sensitivity was shown for the Rapid Polymyxin test $(98.8 \%)$ with a $100 \%$ specificity.

Table 1. Susceptibility category (susceptible or resistant) concordance of different assays compared to reference broth microdilution method. 


\begin{tabular}{|c|c|c|c|c|c|c|c|c|c|}
\hline \multirow{2}{*}{$\begin{array}{l}\text { Method } \\
\text { Specie }\end{array}$} & \multirow{2}{*}{$\begin{array}{l}\text { BDM } \\
\text { S/R }\end{array}$} & \multicolumn{2}{|c|}{ Vitek 21} & \multicolumn{2}{|c|}{ Rapid Polymyxin NP 2} & \multicolumn{2}{|l|}{ UMIC } & \multicolumn{2}{|l|}{ E-test } \\
\hline & & $\mathrm{S} / \mathrm{R}$ & $\begin{array}{l}\% \\
\text { concordance }\end{array}$ & $S / R$ & \% & $S / R$ & \% & $S / R$ & \% Concordance \\
\hline Acinetobacterspp. & $6 / 1$ & $4 / 0$ & 100 & $1 / 1$ & 50 & $6 / 1$ & 100 & $6 / 1$ & 100 \\
\hline C. koseri & $3 / 0$ & $3 / 0$ & 100 & $3 / 0$ & 100 & $3 / 0$ & 100 & $3 / 0$ & 100 \\
\hline E. aerogenes & $1 / 1$ & $1 / 1$ & 100 & $1 / 1$ & 100 & $1 / 1$ & 100 & $1 / 1$ & 100 \\
\hline E. cloacae & $2 / 3$ & $3 / 2$ & 80 & $2 / 3$ & 100 & $2 / 3$ & 100 & $3 / 2$ & 80 \\
\hline E. coli & $13 / 11$ & $13 / 11$ & 100 & $13 / 11$ & 100 & $13 / 11$ & 100 & $13 / 11$ & 100 \\
\hline Hafnia spp. & $0 / 15$ & $1 / 14$ & 93.3 & $0 / 15$ & 100 & $0 / 15$ & 100 & $0 / 15$ & 100 \\
\hline K. oxytoca & $1 / 2$ & $1 / 2$ & 100 & $1 / 2$ & 100 & $1 / 2$ & 100 & $1 / 2$ & 100 \\
\hline K. pneumonaie & $8 / 12$ & $9 / 10$ & 94.7 & $9 / 11$ & 95 & $9 / 11$ & 95 & $9 / 11$ & 95 \\
\hline M. morganii & $0 / 3$ & $0 / 3$ & 100 & $0 / 3$ & 100 & $0 / 3$ & 100 & $0 / 3$ & 100 \\
\hline P. mirabilis & $0 / 2$ & $0 / 2$ & 100 & $0 / 2$ & 100 & $0 / 2$ & 100 & $0 / 2$ & 100 \\
\hline$P$. vulgaris & $0 / 1$ & $0 / 1$ & 100 & $0 / 1$ & 100 & $0 / 1$ & 100 & $0 / 1$ & 100 \\
\hline$P$. aeruginosa & $9 / 1$ & $8 / 0$ & 87.5 & NA & NA & $10 / 0$ & 90 & $10 / 0$ & 90 \\
\hline S. marcescens & $0 / 2$ & $0 / 2$ & 100 & $0 / 2$ & 100 & $0 / 2$ & 100 & $0 / 2$ & 100 \\
\hline Total & $43 / 54$ & $43 / 48$ & 95.6 & $30 / 52$ & 98.8 & $45 / 52$ & 97.9 & $46 / 51$ & 96.9 \\
\hline \multicolumn{10}{|c|}{$B D M$, Broth microdilution; $S$, susceptible; $R$, resistant; $N A$; not applicable } \\
\hline \multicolumn{10}{|c|}{$\begin{array}{l}{ }^{1} A \text { total of } 91 \text { isolates were tested by Vitek method. Isolates not tested by Vitek test, were excluded from the comparison analysis } \\
\text { with broth microdilution. }\end{array}$} \\
\hline
\end{tabular}

Exploring subsets of bacterial isolates, the most robust test compared to the gold standard in Enterobacterales was the UMIC test, with a concordance of $98.7 \%$. The Rapid Polymyxin test also achieved a high concordance level (98.5\%), whereas the Vitek 2 and the E-test were concordant only in $96.2 \%$ of the tested isolates in both tests. In susceptibility testing for non-fermenting bacteria, the highest concordance to broth microdilution was the UMIC (94.1\%) and E-test (94.4\%) followed by Vitek (90.9\%). The rapid polymyxin NP test is specifically designed to detect polymyxin resistance among Enterobacteriaceae(32). The susceptibility concordance with BDM was high for the subset of Enterobacterales isolates included in this study (98.5\%). However, and as expected, the performance for non-fermenting bacteria was poor, reaching a concordance percentage of only $50 \%$. No differences were observed in the capability to detect resistant isolates in non-fermenting and fermenting Enterobacterales between the different assays used in this study (Additional file 3).

MIC concordance of the different assays compared to the gold standard is shown in Table 2. Concordance was established as the same MIC value or \pm 1 titre difference as that of the gold standard. All intrinsic species were excluded from the analysis since no MIC value was obtained from the reference standard method as they were automatically considered as resistant isolates. The highest concordance was achieved with UMIC test (80.4\%), followed by Vitek 2 (72.5\%). The lowest concordance was found for colistin MIC strip of $62.9 \%$.

Table 2. MIC concordance of different assays compared to the reference broth microdilution method. 


\begin{tabular}{llllllll} 
Method & BDM & VITEK & UMIC & & E-test \\
\hline Specie & $\begin{array}{l}\text { No. } \\
\text { Isolates } \\
\text { tested }\end{array}$ & $\begin{array}{l}\text { No. } \\
\text { Isolates } \\
\text { tested }\end{array}$ & $\begin{array}{l}\text { No. of } \\
\text { concordant } \\
\text { isolates [\%] }\end{array}$ & $\begin{array}{l}\text { No. } \\
\text { Isolates } \\
\text { tested }\end{array}$ & $\begin{array}{l}\text { No. of } \\
\text { concordant } \\
\text { isolates [\%] }\end{array}$ & $\begin{array}{l}\text { No. } \\
\text { Isolates } \\
\text { tested }\end{array}$ & $\begin{array}{l}\text { No. of concordant } \\
\text { isolates [\%] }\end{array}$ \\
\hline $\begin{array}{l}\text { Acinetobacter } \\
\text { spp. }\end{array}$ & 7 & 4 & $4[100.0]$ & 7 & $7[100.0]$ & 7 & $6[85.7]$ \\
\hline C. koseri & 3 & 3 & $3[100.0]$ & 3 & $2[66.7]$ & 3 & $2[66.7]$ \\
\hline E. aerogenes & 2 & 2 & $1[50.0]$ & 2 & $1[50.0]$ & 2 & $2[100.0]$ \\
\hline E. cloacae & 5 & 5 & $3[60.0]$ & 5 & $5[100.0]$ & 5 & $3[60.0]$ \\
\hline E. coli & 24 & 24 & $17[70.8]$ & 24 & $19[79.2]$ & 24 & $13[54.2]$ \\
\hline Hafnia spp. & 15 & 15 & $14[93.3]$ & 15 & $14[93.3]$ & 15 & $13[86.7]$ \\
\hline K. oxytoca & 3 & 3 & $1[33.3]$ & 3 & $3[100.0]$ & 3 & $1[33.3]$ \\
\hline $\begin{array}{l}\text { K. } \\
\text { pneumoniae }\end{array}$ & 20 & 19 & $16[84.2]$ & 20 & $17[85.0]$ & 20 & $13[65.0]$ \\
\hline P. aeruginosa & 10 & 8 & $7[87.5]$ & 10 & $10[100.0]$ & 10 & $8[80.0]$ \\
\hline Total & 97 & 91 & $66[72.5]$ & 97 & $78[80.4]$ & 97 & $61[62.9]$ \\
\hline
\end{tabular}

BDM, Broth microdilution method

${ }^{1}$ Concordance was considered as the same MIC value or as one titre difference to that of the reference value

Additionally, for Enterobacterales species the most concordant test to the gold standard was UMIC (76.25\%), followed by VITEK (69.62\%) and E-test (58.75\%). Similarly, the highest concordance for non-fermenting bacteria was found in the UMIC test (100\%). The Vitek 2 test and the E-test achieved a concordance to the gold standard of $91.67 \%$ and $82.35 \%$, respectively. Figure 2 shows the MIC distribution of the BDM vs. each method and the MIC distribution for all isolates.

Heterogenous molecular causes of colistin resistance.

MLST sequence type (ST) designation and core genome MLST (cgMLST) analysis were used to establish the diversity between isolates. The STs of isolates for which species MLST schemes exist was determined (Additional file 4). cgMLST could only be performed on species with more than two isolates. The diversity within E. coli and $K$. pneumoniae are shown in Figure 3. Isolates were genomically diverse in the cgMLST with the exception of isolates from the same patients (indicated with * in the figures): in some cases multiple isolates belonged to the same ST, such as K. pneumoniae ST512 ( $n=7), K$. pneumoniae ST1825 (n=2), E. coli ST73 $(n=3)$, and E. coli ST156 $(n=2)$.

Genes encoding colistin resistance were identified first by comparing genome assemblies against known databases. This identified $m c r-$ 1 in four isolates (700099-17, NCTC-13846, 719645-16 and 705498-12) and mcr-2 in one isolate (KP-37), but these results did not explain all the phenotypic resistance.

Individual genomic analysis of the underlying colistin resistance mechanisms was performed, looking at genes previously described as being involved in colistin resistance. These genes were extracted from the genome and compared between sensitive and resistant isolates. This could only be performed on isolates within species with sufficient numbers of each, namely $K$. pneumoniae $(\mathrm{n}=20)$ and $E$. coli $(\mathrm{n}=24)$. The nucleotide sequences and derived protein sequences from $p h o P Q$ and $p m r C A B$ in both species, and additionally $m g r B$ in K. pneumoniae isolates were compared between resistant and susceptible isolates (Additional files 5, 6 and 7). Variations unique to the resistant isolates are described in Table 3.

None of the analysed $K$. pneumoniae isolates were carriers of $\mathrm{mcr}$ genes. A key finding within $K$. pneumoniae isolates was the presence of mutations in $\mathrm{mgr} B$ causing amino acid substitutions, premature stop codons, or termination resulting from insertion sequences ISEcp1 (IS138 family) and ISkpn26 (IS5 family). This applied to all resistant isolates with MIC values of $\geq 16 \mathrm{mg} / \mathrm{L}$, whereas sensitive isolates have intact versions of $m g r B$. Further mutations leading to amino acid substitutions were found in other genes: the isolate with the highest MIC ( $\geq 64 \mathrm{mg} / \mathrm{L}$ ) possesses additional amino acid substitutions in the PhoQ, PmrA, and PmrB proteins compared to those from sensitive isolates. Similarly, two isolates with MIC of $32 \mathrm{mg} / \mathrm{L}$ have amino acid substitutions in the PmrA and/or PmrC protein in 
addition to MgrB. Two resistant isolates with MICs of $4 \mathrm{mg} / \mathrm{ml}$ and $8 \mathrm{mg} / \mathrm{L}$ have an unaltered $m g r B$ gene but possesses nonsynonymous mutations in the $p m r A$ and/or pmrC genes.

Only four of the resistant $E$. coli isolates, with MICs between 4-8 mg/ml were carriers of the $m c r-1.1$ gene. Gene comparisons within $E$. coli showed that those with a MIC of $16 \mathrm{mg} / \mathrm{ml}$ have amino acid changes in the PmrB protein sequence, whereas the isolates with a lower MIC (4-8mg/ml) possess PmrB identical to those in sensitive isolates. In one isolate with a MIC of $16 \mathrm{mg} / \mathrm{ml}$, the $p m r A$ and $p m r B$ genes were not found within the genome assembly and therefore could not be analysed for mutations. Two isolates with a MIC of 8 mg/ml that did not harbour $m c r$ genes had an unaltered PmrB protein sequence compared to the susceptible isolates but had amino acid substitutions in the protein sequences of PmrA or PmrC.

All the $A$. bereziniae isolates $(\mathrm{n}=4)$ were isolated from the same patient, and were subjected to analysis of $p m r A B$ and $p h o P Q$ genes, as well as $I p x A$, I $P x D, I p \times C$ genes since mutations in the latter genes produce a total loss of lipid $A$ leading to colistin resistance in Acinetobacter spp. (5). The single resistant isolate (MIC 64mg/ml) had a mutation causing amino acid change Q242R in PmrB. No mutations were found in the other genes assessed.

Table 3. Mutations in associated colistin-resistance proteins in E. coli, K. pneumoniae and A. berziniae resistant isolates. 


\begin{tabular}{|c|c|c|c|c|c|c|c|c|c|}
\hline \multirow[t]{2}{*}{ Species } & \multirow[t]{2}{*}{ Isolate } & \multirow[t]{2}{*}{$\begin{array}{l}\text { MIC } \\
(\mathrm{mg} / \mathrm{l})^{1}\end{array}$} & \multicolumn{6}{|c|}{ Amino acid change } & \multirow{2}{*}{$\begin{array}{l}\text { Plasmid mediated } \\
\text { resistance } \\
\text { mcr }\end{array}$} \\
\hline & & & MgrB & PmrB & PmrA & PmrC & PhoP & PhoQ & \\
\hline \multirow{13}{*}{$\begin{array}{l}\text { K. } \\
\text { pneumoniae }\end{array}$} & $404507-16$ & $\geq 64$ & D31N & L213M & A41T & & & S288N & \\
\hline & $4002006-2$ & 32 & $\mathrm{~K} 3^{*}$ & & & & & & \\
\hline & 16003084 & 32 & C28S & & A217V & G25S & & & \\
\hline & 20038016 & 32 & C39Y & & & & & & \\
\hline & \multirow[t]{2}{*}{$808927-16$} & \multirow[t]{2}{*}{32} & \multirow[t]{2}{*}{ L8* } & & & $\mathrm{R} 152 \mathrm{H}$ & & & \\
\hline & & & & & & D477N & & & \\
\hline & 26048671 & 32 & $\begin{array}{l}141^{*} \\
(\text { ISKpn26) }\end{array}$ & & & & & & \\
\hline & $800138-16$ & 32 & $\begin{array}{l}141^{*} \\
(\text { ISEcp } 1)^{3}\end{array}$ & & & & & & \\
\hline & 401433-14 & 16 & Q30* & & & & & & \\
\hline & $802208-17$ & 16 & $\mathrm{~L} 4^{*}$ & & D149E & & & & \\
\hline & 187701876 & 16 & C39G & & & & & & \\
\hline & 19852760 & 8 & & & A217V & G25S & & & \\
\hline & 809156-16 & 4 & & & D149E & & & & \\
\hline \multirow[t]{12}{*}{ E. coli } & $721296-16$ & 16 & & P97L & & $\mathrm{C} 27 \mathrm{Y}$ & & & \\
\hline & $700455-17$ & 16 & & L197D & & N12D & & & \\
\hline & 709006-16 & 16 & & E169K & & & & & \\
\hline & 705963-16 & 16 & & $N^{2}{ }^{2}$ & $N^{2}{ }^{2}$ & $N^{2}$ & & & \\
\hline & 700099-17 & 8 & & & & & & & mer 1.1 \\
\hline & 706090-16 & 8 & & & $\mathrm{R} 81 \mathrm{H}$ & Q479E & & & \\
\hline & NCTC-13846 & 8 & & & & & & L467M & $\operatorname{mor} 1.1$ \\
\hline & \multirow{2}{*}{$\begin{array}{l}\text { KP-37-MCR-2- } \\
18\end{array}$} & \multirow[t]{2}{*}{8} & & & & $\mathrm{C} 27 \mathrm{Y}$ & & & \multirow[t]{2}{*}{ mor 2} \\
\hline & & & & & & L74I & & & \\
\hline & 707671-17 & 8 & & & & $\mathrm{C} 27 \mathrm{Y}$ & & & \\
\hline & 719645-16 & 8 & & & & & & & $\operatorname{mor} 1.1$ \\
\hline & 705498-12 & 4 & & & & & & & $\operatorname{mor} 1.1$ \\
\hline A. bereziniae & $502814-14$ & $\geq 64$ & & Q242R & & & & & \\
\hline \multicolumn{10}{|c|}{${ }^{7} \mathrm{MIC}$ values obtained by the reference broth microdilution method } \\
\hline${ }^{2} N F, p m r A B n$ & found in asse & & & & & & & & \\
\hline
\end{tabular}

\section{Discussion}

We compared the performance of four commercial assays for colistin susceptibility testing to the broth microdilution gold standard. We used a panel of different bacterial species to reflect common situations found in routine diagnostics. 
Numerous comparative studies dealing with colistin susceptibility testing have been published. Several studies find the highest rate of very major errors (VMEs), considered as discrepancy in the susceptibility category between a commercial kit and the reference method, with E-test colistin $(6,33,34)$, which was also confirmed with our study. Vitek 2 has been reported as reliable in some studies, but not in others (35-37). In our study, the rapid Polymyxin NP Test showed the highest concordance with the gold standard in the susceptibility category agreement. However, in our experience, the main drawback is lack of MIC values and difficulties in interpretation of the colorimetric test. The UMIC test was easy to perform and showed highest categorical and MIC agreement. This has however not been confirmed in other studies. Due to the high performance of the UMIC assay, this assay was established into routine diagnostics at the University Hospital Basel.

For this study we included 54 (56\%) colistin resistant isolates, of which 23 (43\%) were intrinsically resistant whereas 31 (57\%) had acquired resistance mechanism. All the isolates were collected during 2017 but the susceptibility or resistance to colistin was determined following the EUCAST Version 10.02020 (23). The breakpoints for Enterobacterales in the 2020 EUCAST version remained unaltered compared to the 2017 version 7.1 (38) that applied when the isolates were collected, whereas the breakpoint for Pseudomonas spp. changed to lower MIC values ( $\leq 2 \mathrm{mg} / \mathrm{l}, \mathrm{R}>2 \mathrm{mg} / \mathrm{l})$. However, these cut off changes did not affect the percentage of resistant isolates included in this study. Moreover, four E. coli isolates were carriers of the $\mathrm{mcr}-1 \mathrm{gene}$, and one isolate was positive for the $m c r-2$. As expected, none of $\mathrm{mcr}$-positive isolates displayed a high MIC value, considered in this study as $\geq 16 \mathrm{mg} / \mathrm{ml}$.

Several chromosomal mutations have been reported to be linked to colistin resistance in various species $(4,5,11,39,40)$. Most of the reported mutations have been found in genes involved in signalling pathways that lead to modification or loss of lipid A from the LPS. Mutations in genes related to efflux pumps have also been described $(41,42)$. The most commonly reported colistin-related mutations are encountered in the $p m r A / p m r B$ and $p h o P / p h o Q$ genes encoding two-component systems in several Gram-negative bacteria such as $E$. coli, K. pneumoniae, $P$. aeruginosa and $A$. baumanii (5). The $m g r B$ gene encodes a negative regulator of the PhoPQ system. $m g r B$ inactivation or disruption have been associated with colistin resistance in $K$. pneumonia $(15,43)$. Although these are the most commonly described genes the chromosomal mechanisms leading to resistance are highly diverse and involve numerous and different mutations and genes (5).

In this study, we found that all the $K$. pneumoniae isolates displaying a high MIC ( $\geq 16 \mathrm{mg} / \mathrm{ml})$ had a disrupted or altered $\mathrm{mgr} B$ gene and an altered protein sequence, whereas isolates with MICs $£ 8 \mathrm{mg} / \mathrm{ml}$ displayed a wild type $m g r B$. Some of the amino acid alterations encountered in MgrB during this study (truncations at K3 and Q30, substitutions at C28S, C39Y/G and disruption of the gene by ISKpn26 and ISEcP1) have been described in other studies $(43,44)$. However, to our best knowledge, the truncations at L4 and L8, and the D31N amino acid change are novel to this study. Noteworthy, the cgMLST analysis identified several closely related $K$. pneumoniae isolates (ST512) with different susceptibilities to colistin. Within this ST type, all resistant isolates carried mutations in the $m g r B$ gene, leading to altered MgrB protein sequences, and in no other colistin resistance-related protein sequences we analysed. However, these alterations were unique in each isolate and appear to have occurred independently. Additionally, we identified further mutations in the $m g r B$ gene in isolates from other STs, which also led to high MIC values. These results suggest that mutations of $m g r B$ in any of these locations can lead to high colistin resistance in $K$. pneumoniae and that there is not a unique mgrB mutation associated with a specific ST type. The single K. pneumoniae isolate with a $\mathrm{MIC} \geq 64 \mathrm{mg} / \mathrm{ml}$ also carried altered $p m r B$ and $p h o Q$ in addition to an $m g r B$ mutant (causing $\mathrm{D} 31 \mathrm{~N}$ ). The mutation in $p m r B$ (causing L213M) has already been associated with colistin resistance in $K$. pneumoniae (43). This may indicate that association between $p m r B$ and/or $p h o Q$ mutations with $m g r B$ mutations confer a higher resistance than these mutations on their own. Together this data suggests that mutation or inactivation of the $\mathrm{mgr} B$ gene leads to MICs $\geq 16 \mathrm{mg} / \mathrm{ml}$, with further mutations in pathway genes able to synergistically increase MICs further.

Similarly, all the resistant E. coli isolates with MIC $16 \mathrm{mg} / \mathrm{ml}$ carried amino acid changes in PmrB. Of these amino acid substitutions (P97L, E169K and L197D), only that at position 97 has been reported previously, in a clinical colistin resistant isolate from a Lebanese hospital, also displaying a MIC of $16 \mathrm{mg} / \mathrm{ml}$ (45). Although the amino acid change in this position was different to the one in our study. As far as we are aware, the other two pmrB alterations have not been described, although other alterations within the same domains, namely the HAMP domain (position 92-144) and histidine-kinase domain (position 145-205) have been reported (5,14,39). For one high resistant isolate (705963-16), the pmrCAB operon was not found in the genome assembly and, therefore, mutations within these genes could not be determined. Whether this operon has been deleted or whether this is a sequencing artefact is unclear. Further amino acid changes were found in PmrA, PmrC and PhoQ in isolates with MIC between 4-8mg/ml, suggesting that alterations in these proteins may confer a lower level of resistance. Of these, only the amino acid change at position 81 in PmrA from E. coli has been previously characterized, in an isolate from swine origin and with a colistin MIC of $4 \mathrm{mg} / \mathrm{ml}$ (18). In this published case, it was not determined whether the mutation in PmrA was the sole cause of colistin resistance, as other mutations were also identified within this isolate.

Page 9/16 
Total loss of lipid A of the LPS by alteration of $I x p A$, IxpC and IpxD genes has been described as a colistin resistance mechanism in Acinetobacter baumannii (5). Mutations in pmrAB also lead to colistin resistance in this species $(19,46,47)$. A published comparison between susceptible and resistant $A$. baumannii isolates after in vivo exposure in three different patients found different but unique mutations in $p m r B$ that led to resistance levels of $16 \mathrm{mg} / \mathrm{ml}$ (47). Similarly, we found that the only difference between the susceptible and resistant $A$. bereziniae isolates from the same patient was a single amino acid change (Q242R) in the PmrB protein. Interestingly, the resistant isolate was highly resistant to colistin (MIC $\geq 64 \mathrm{mg} / \mathrm{ml}$ ). Detection of an alteration at this same position has so far not been reported. This may suggest that alterations in PmrB in Acinetobacter spp. and/or the specific PmrB alteration encountered in this study (Q242R) are putative mechanisms that confer high levels colistin resistance.

Typing isolates shows that resistance can occur in diverse isolates within the species we analysed. Our data also suggests the impact of selective pressure, with stochastic presence of resistance throughout the phylogenies, and resistant isolates of $A$. berziniae and $K$. pneumoniae (ST512) closely related to sensitive isolates.

This study has several limitations. Only small number of isolates $(n=10)$ showed MICs close to the breakpoint. Future studies should include more isolates close to the breakpoint. Secondly, we were not able to investigate putative resistance mechanisms in all species due to the low number of isolates. Thirdly, we have investigated putative resistance mechanisms in diverse clinical isolates and have not confirmed the effects of the observed mutations in isogenic backgrounds.

In summary, in our clinical setup MIC values provide important information. The UMIC assay provided the highest concordance on MIC values with the reference method. For a categorical assessment the Rapid Polymyxin NP test provided highly concordant results. Our genetic study showed highly heterogenous reasons for resistance. Whereas some resistant assays may affect the MIC only weakly, sensitive and precise phenotypic assays are important in routine diagnostics.

\section{Abbreviations}

LPS: Lipopolysaccharide, L-Ara-4N: 4-amino-4-deoxy-L-arabinose, pEtN: phosphoethanolamine, MIC: Minimum inhibitory concentration, CPS: Capsular polysaccharide, pmrA: two-component regulator system response regulator PmrA, pmrB: two-component regulator system response regulator PmrB, phoP: response regulator in two-component regulatory system with PhoQ, phoQ: two-component system sensor histidine kinase PhoQ, mgrB: PhoP/PhoQ regulator MgrB, mcr. mobilized colistin resistance, IpxA: UDP-N-acetylglucosamine acyltransferase, IpxC: UDP-3-O-acyl-N-acetylglucosamine deacetylase, IpxD: UDP-3-O-(3-hydroxymyristoyl)glucosamine Nacyltransferase, EUCAST: European Committee on Antimicrobial Susceptibility Testing, BDM: Broth Microdilution, MLST: Multilocus sequence typing, cgMLST: core genome Multilocus sequence typing, NCBI: National Center for Biotechnology Information, CARD AMR: The Comprehensive Antibiotic Resistance Database, ANI: Average Nucleotide Identity, MALDI-TOF MS: Matrix-Assisted Laser Desorption Ionization T-Of-Flight Mass Spectrometry, VME: Very Major Errors, HAMP: histidine kinases, adenylyl cyclases, methyl binding proteins, and phosphatases.

\section{Declarations}

\section{Ethics approval and consent to participate.}

This study was conducted as a quality control study and for assay development - no patient specific data was used. For this reason, no ethical approval was necessary.

\section{Consent for publication.}

All authors have read the publication and agree with its content.

\section{Availability of data and materials.}

The dataset(s) supporting the conclusions of this article are included within the article and its additional files. Additional files are: Additional file 1 - Species distribution.docx showing the Species distribution as determined by MALDI-TOF MS. A total of 97 clinical isolates were collected from the Basel University Hospital (Basel, Switzerland), Cantonal Hospital Luzerne (Luzerne, Switzerland) and Laboratory Viollier (Allschwil, Switzerland); Additional file 2 Different phenotypic assays used for colistin susceptibility testing: UMIC, ETest and Rapid Polymyxin NP Test.docx showing example images of the phenotypic assays using in this study; Additional file 3 Susceptibility category (susceptible or resistant) concordance of different assays compared to the reference broth microdilution method 
in Enterobacterales species and non-fermenting bacteria.xlsx showing the percentage of concordance for different test in Enterobacterales and non-fermenting bacteria; Additional file 4 - species identification by MALDI-TOF MS and rMLST from whole genome sequencing data.docx showing the difference in species identification by MALDI-TOF MS and rMLST as well as the ST types of every isolate included in this study; Additional file 5 - Klebsiella pneumoniae protein sequence alignments.pdf showing the protein sequences alignments of all colistin resistance-related proteins analyzed in this study; Additional file 6 - Escherichia coli protein sequence alignments.pdf showing the protein sequences alignments of all colistin resistance-related proteins analyzed in this study; Additional file 7 - Acinetobacter bereziniae protein sequence alignments.pdf showing the protein sequences alignments of all colistin resistance-related proteins analyzed in this study.

\section{Competing interests}

The authors declare that they have no competing interests.

\section{Funding}

The study was funded by development funding to Adrian Egli at the University Hospital Basel.

\section{Authors contributions}

AE designed the study and supervised the phenotypical part. DAT and HMBSS performed whole genome sequencing data analysis and drafted the first version of the manuscript. All authors revised the manuscript and provided feedback.

\section{Acknowledgements}

We thank Aline Cuenod (University of Basel) for assistance with Klebsiella species identification. We thank Dr. Janina E. Linnik (ETH Zurich) for visualization of MIC distributions. We thank Christine Kiessling, Magdalena Schneider, Elisabeth Schultheiss, Clarisse Straub, and Rosa-Maria Vesco (University Hospital Basel) for excellent technical assistance in whole genome sequencing. Assemblies and searches were performed at sciCORE (http://scicore.unibas.ch/) scientific computing centre at the University of Basel. We thank Prof. Herbert Hächler (NENT, University of Zurich) for reference measurements of colistin minimal inhibitory concentrations. We thank the Luzener Kantosspital for providing strains also included in this study.

\section{References}

1. Medicines Agency E. Updated advice on the use of colistin products in animals within the European Union: development of resistance and possible impact on human and animal health [Internet]. 2016 [cited 2020 May 19]. Available from: .

2. Ecdc. PRESS RELEASE Resistance to last-line antibiotics continues to cause concern in Europe [Internet]. 2014 [cited 2020 May 19]. Available from: .

3. Nordmann P, Naas T, Poirel L. Global spread of carbapenemase producing Enterobacteriaceae. Emerg Infect Dis. 2011;17(10):17918.

4. Aghapour Z, Gholizadeh P, Ganbarov K, Bialvaei AZ, Mahmood SS, Tanomand A, et al. Molecular mechanisms related to colistin resistance in enterobacteriaceae. Infect Drug Resist. 2019;12:965-75.

5. Poirel L, Jayol A, Nordmanna P. Polymyxins. Antibacterial activity, susceptibility testing, and resistance mechanisms encoded by plasmids or chromosomes. Vol. 30, Clinical Microbiology Reviews. American Society for Microbiology; 2017. pp. 557-96.

6. Chew KL, La M, Van, Lin RTP, Teo JWP. Colistin and polymyxin B susceptibility testing for carbapenem-resistant and mcr-positive enterobacteriaceae: Comparison of Sensititre, MicroScan, Vitek 2, and Etest with broth microdilution. J Clin Microbiol. 2017 Sep 1;55(9):2609-16.

7. Deris ZZ, Akter J, Sivanesan S, Roberts KD, Thompson PE, Nation RL, et al. A secondary mode of action of polymyxins against Gram-negative bacteria involves the inhibition of NADH-quinone oxidoreductase activity. [cited 2020 May 12]; Available from: http://www.nature.com/authors/editorial_policies/license.html\#terms.

8. Li J, Nation RL, Milne RW, Turnidge JD, Coulthard K. Evaluation of colistin as an agent against multi-resistant Gram-negative bacteria [Internet]. Vol. 25, International Journal of Antimicrobial Agents. Elsevier BV. 2005 [cited 2020 May 12]. p. 11-25. Available from: http://www.ncbi.nlm.nih.gov/pubmed/15620821. 
9. El-Sayed Ahmed MAE-G, Zhong L-L, Shen C, Yang Y, Doi Y, Tian G-B. Colistin and its role in the Era of antibiotic resistance: an extended review (2000-2019). Emerg Microbes Infect [Internet]. 2020 Jan 1 [cited 2020 May 27];9(1):868-85. Available from: https://www.tandfonline.com/doi/full/10.1080/22221751.2020.1754133.

10. Jayol A, Saly M, Nordmann P, Ménard A, Poirel L, Dubois V. Hafnia, an enterobacterial genus naturally resistant to colistin revealed by three susceptibility testing methods. J Antimicrob Chemother. 2017;72(9):2507-11.

11. Mlynarcik P, Kolar M. Molecular mechanisms of polymyxin resistance and detection of mcr genes. Biomed Pap [Internet]. 2019;163(1):28-38. Available from: https://doi.org/10.5507/bp.2018.070.

12. Gharaibeh $\mathrm{MH}$, Shatnawi SQ. An overview of colistin resistance, mobilized colistin resistance genes dissemination, global responses, and the alternatives to colistin: A review. Vet World. 2019;12(11):1735-46.

13. Colistin M, Genes R. Global Burden of Colistin-Resistant Bacteria: Mobilized Colistin Resistance Genes Study. 2019;1-18.

14. Olaitan AO, Morand S, Rolain JM. Mechanisms of polymyxin resistance: Acquired and intrinsic resistance in bacteria. Front Microbiol. 2014;5(NOV):1-18.

15. Poirel L, Jayol A, Bontron S, Villegas MV, Ozdamar M, Türkoglu S, et al. The mgrB gene as a key target for acquired resistance to colistin in Klebsiella pneumoniae. J Antimicrob Chemother. 2015;70(1):75-80.

16. De Majumdar S, Yu J, Fookes M, McAteer SP, Llobet E, Finn S, et al. Elucidation of the RamA Regulon in Klebsiella pneumoniae Reveals a Role in LPS Regulation. PLoS Pathog. 2015;11(1):1-22.

17. Castanheira M, Castanheira M, Doyle T, Davis AP, Deshpande LM, Mendes RE. Disruption of mgrB and Alterations on pmrB Are Most Common Resistance Mechanisms among Colistin-Resistance among Klebsiella pneumoniae from a Global Surveillance Program [Internet]. [cited 2020 May 19]. Available from: http://www.eucast.org/fileadmin/src/media.

18. Quesada A, Concepción Porrero M, Téllez S, Palomo G, García M, Domínguez L. Polymorphism of genes encoding PmrAB in colistinresistant strains of Escherichia coli and Salmonella enterica isolated from poultry and swine. J Antimicrob Chemother. 2015;70(1):71-4.

19. Boinett CJ, Cain AK, Hawkey J, Hoang NT, Do, Khanh NNT, Thanh DP, et al. Clinical and laboratory-induced colistin-resistance mechanisms in acinetobacter baumannii. Microb Genomics. 2019;5(2).

20. Turlej-Rogacka A, Xavier BB, Janssens L, Lammens C, Zarkotou O, Pournaras S, et al. Evaluation of colistin stability in agar and comparison of four methods for MIC testing of colistin. Eur J Clin Microbiol Infect Dis. 2018 Feb 1;37(2):345-53.

21. EUCAST. Guidance documents [Internet]. [cited 2020 May 19]. Available from: https://www.eucast.org/ast_of_bacteria/guidance_documents/.

22. Xavier BB, Lammens C, Ruhal R, Malhotra-Kumar S, Butaye P, Goossens H, et al. Identification of a novel plasmid-mediated colistinresistance gene, mcr-2, in Escherichia coli, Belgium, june 2016. Eurosurveillance [Internet]. 2016 Jul 7 [cited 2021 Mar 30];21(27). Available from: https://pubmed.ncbi.nlm.nih.gov/27416987/.

23. EUCAST clinical breakpoints - bacteria v 5.0. (until Dec 31, 2015) [Internet]. [cited 2020 Jul 13]. Available from: https://www.eucast.org/fileadmin/src/media/PDFs/EUCAST_files/Breakpoint_tables/v_5.0_Breakpoint_Table_01.pdf.

24. Bolger AM, Lohse M, Usadel B. Trimmomatic: a flexible trimmer for Illumina sequence data. Bioinformatics [Internet]. 2014 Apr 1;30(15):2114-20. Available from: https://doi.org/10.1093/bioinformatics/btu170.

25. Wick RR, Judd LM, Gorrie CL, Holt KE. Unicycler: Resolving bacterial genome assemblies from short and long sequencing reads. Phillippy AM, editor. PLOS Comput Biol [Internet]. 2017 Jun 8 [cited 2020 May 27];13(6):e1005595. Available from: https://dx.plos.org/10.1371/journal.pcbi.1005595.

26. Seemann T. Prokka: rapid prokaryotic genome annotation. Bioinformatics [Internet]. 2014 Mar 18;30(14):2068-9. Available from: https://doi.org/10.1093/bioinformatics/btu153.

27. GitHub - tseemann/abricate. Mass screening of contigs for antimicrobial and virulence genes [Internet]. [cited 2020 May 27]. Available from: https://github.com/tseemann/abricate.

28. Ribosomal Multilocus Sequence. Typing (rMLST) - PubMLST.org [Internet]. [cited 2020 May 27]. Available from: https://pubmlst.org/rmlst/.

29. cgMLST.org. Nomenclature Server [Internet]. [cited 2020 May 27]. Available from: https://www.cgmlst.org/ncs.

30. Carver T, Harris SR, Berriman M, Parkhill J, McQuillan JA. Artemis: an integrated platform for visualization and analysis of highthroughput sequence-based experimental data. Bioinformatics [Internet]. 2011 Dec 22;28(4):464-9. Available from: https://doi.org/10.1093/bioinformatics/btr703.

Page 12/16 
31. Jalview Version 2-a Multiple Sequence Alignment editor. and Analysis Workbench - PubMed [Internet]. [cited 2020 May 27]. Available from: https://pubmed.ncbi.nlm.nih.gov/19151095/.

32. Rapid Polymyxin ${ }^{T M}$. NP - ELITechGroup: In Vitro Diagnostic Equipment \& Reagents [Internet]. [cited 2021 Apr 23]. Available from: https://www.elitechgroup.com/product/rapid-polymyxinnp\#tab-features.

33. Arroyo LA, García-Curiel A, Pachón-Ibañez ME, Llanos AC, Ruiz M, Pachón J, et al. Reliability of the E-test method for detection of colistin resistance in clinical isolates of Acinetobacter baumannii. J Clin Microbiol [Internet]. 2005 Feb [cited 2020 Jul 13];43(2):903-5. Available from: /pmc/articles/PMC548043/?report = abstract.

34. Matuschek E, Åhman J, Webster C, Kahlmeter G. Antimicrobial susceptibility testing of colistin - evaluation of seven commercial MIC products against standard broth microdilution for Escherichia coli, Klebsiella pneumoniae, Pseudomonas aeruginosa, and Acinetobacter spp. Clin Microbiol Infect [Internet]. 2018 Aug 1 [cited 2020 Jul 13];24(8):865-70. Available from: https://pubmed.ncbi.nlm.nih.gov/29221995/.

35. Dafopoulou K, Zarkotou O, Dimitroulia E, Hadjichristodoulou C, Gennimata V, Pournaras S, et al. Comparative Evaluation of Colistin Susceptibility Testing Methods among Carbapenem-Nonsusceptible Klebsiella pneumoniae and Acinetobacter baumannii Clinical Isolates. 2015.

36. Chew KL, La M, Van, Lin RTP, Teo JWP. Colistin and polymyxin B susceptibility testing for carbapenem-resistant and mcr-positive enterobacteriaceae: Comparison of Sensititre, MicroScan, Vitek 2, and Etest with broth microdilution. J Clin Microbiol [Internet]. 2017 Sep 1 [cited 2020 Jul 13];55(9):2609-16. Available from: https://pubmed.ncbi.nlm.nih.gov/28592552/.

37. De Angelis G, Posteraro B, Menchinelli G, Marzia Liotti F, Spanu T, Sanguinetti M. Antimicrobial susceptibility testing of pathogens isolated from blood culture: a performance comparison of Accelerate Pheno TM and VITEK V R 2 systems with the broth microdilution method.

38. EUCAST. Previous versions of documents [Internet]. [cited 2021 Mar 31]. Available from: https://www.eucast.org/ast_of_bacteria/previous_versions_of_documents/.

39. Bialvaei AZ, Samadi Kafil H. Colistin, mechanisms and prevalence of resistance. Curr Med Res Opin [Internet]. 2015 Apr 1 [cited 2020 May 12];31(4):707-21. Available from: http://www.ncbi.nlm.nih.gov/pubmed/25697677.

40. Berglund B. Acquired Resistance to Colistin via Chromosomal And Plasmid-Mediated Mechanisms in Klebsiella pneumoniae. Infect Microbes Dis [Internet]. 2019;1(1):10-9. Available from: http://journals.Iww.com/10.1097/IM9.0000000000000002.

41. Sundaramoorthy NS, Suresh P, Selva Ganesan S, GaneshPrasad AK, Nagarajan S. Restoring colistin sensitivity in colistin-resistant E. coli: Combinatorial use of MarR inhibitor with efflux pump inhibitor. Sci Rep [Internet]. 2019 Dec 1 [cited 2020 Jul 13];9(1). Available from: https://pubmed.ncbi.nlm.nih.gov/31882661/.

42. Lin MF, Lin YY, Lan CY. Contribution of EmrAB efflux pumps to colistin resistance in Acinetobacter baumannii. J Microbiol [Internet]. 2017 Feb 1 [cited 2020 Jul 13];55(2):130-6. Available from: https://pubmed.ncbi.nlm.nih.gov/28120193/.

43. Castanheira M, Castanheira M, Doyle T, Davis AP, Deshpande LM, Mendes RE. Disruption of mgrB and Alterations on pmrB Are Most Common Resistance Mechanisms among Colistin-Resistance among Klebsiella pneumoniae from a Global Surveillance Program [Internet]. [cited 2020 May 14]. Available from: http://www.eucast.org/fileadmin/src/media.

44. Jayol A, Nordmann P, Lehours P, Poirel L, Dubois V. Comparison of methods for detection of plasmid-mediated and chromosomally encoded colistin resistance in Enterobacteriaceae. Clin Microbiol Infect [Internet]. 2018;24(2):175-9. Available from: https://doi.org/10.1016/j.cmi.2017.06.002.

45. Nawfal Dagher T, Al-Bayssari C, Chabou S, Baron S, Hadjadj L, Diene SM, et al. Intestinal Carriage of Colistin Resistant Enterobacteriaceae at Saint Georges Hospital in Lebanon. J Glob Antimicrob Resist. 2019 Dec 12.

46. Ko KS, Choi Y, Lee J-Y. Old drug, new findings: colistin resistance and dependence of Acinetobacter baumannii. Precis Futur Med. 2017;1(4):159-67.

47. Marano V, Marascio N, Pavia G, Lamberti AG, Quirino A, Musarella R, et al. Identification of pmrB mutations as putative mechanism for colistin resistance in A. baumannii strains isolated after in vivo colistin exposure. Microb Pathog [Internet]. 2020;142(November 2019):104058. Available from: https://doi.org/10.1016/j.micpath.2020.104058.

\section{Figures}




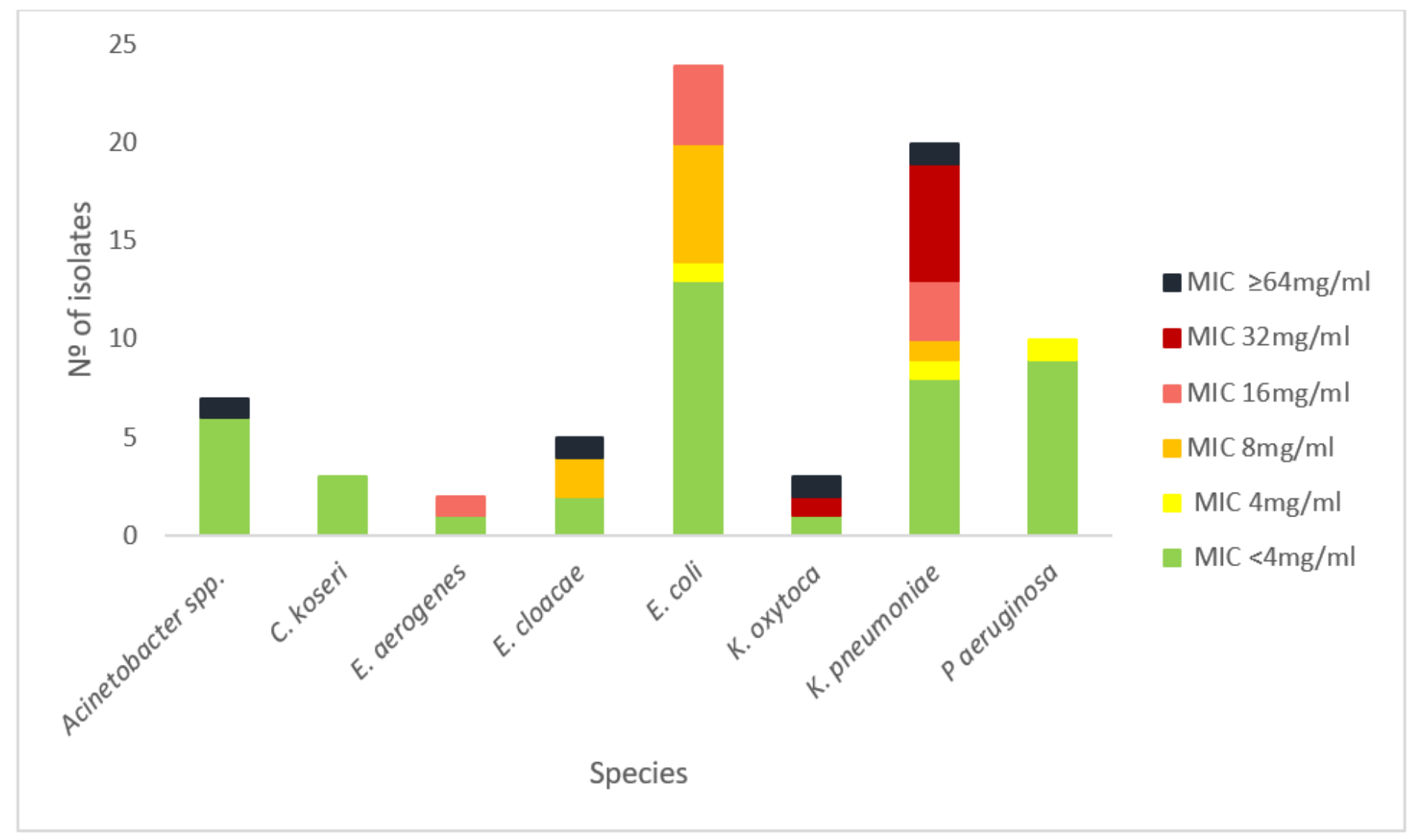

Figure 1

Colistin resistance in non-intrinsically resistant bacteria. MICs were determined by broth microdilution methods (BDM) and interpreted according to EUCAST breakpoints (Version 10.0, 2020).

Etest

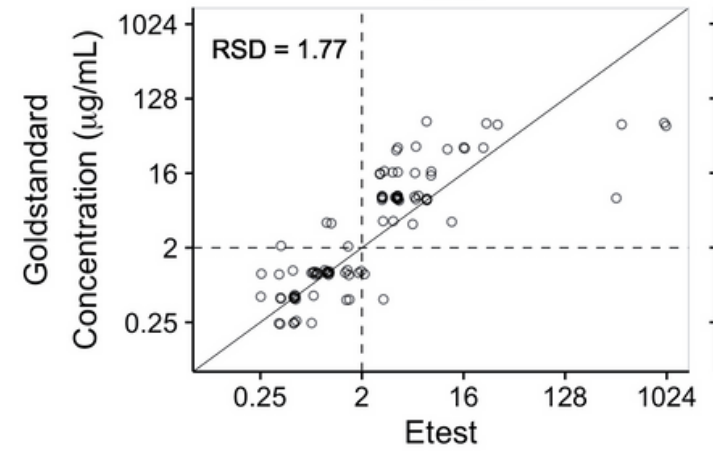

Concentration $(\mu \mathrm{g} / \mathrm{mL})$

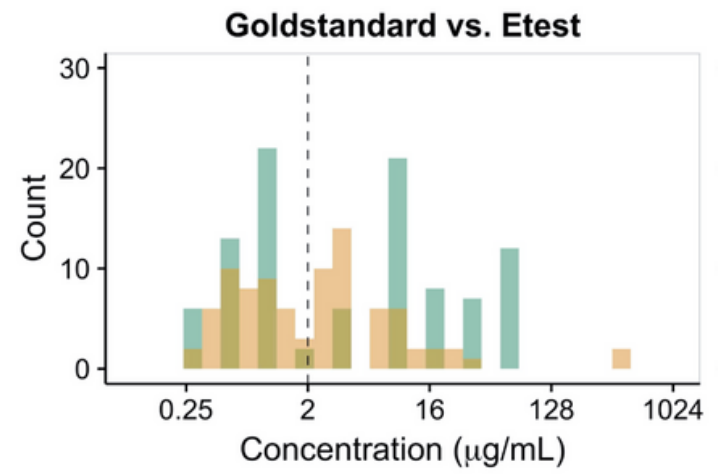

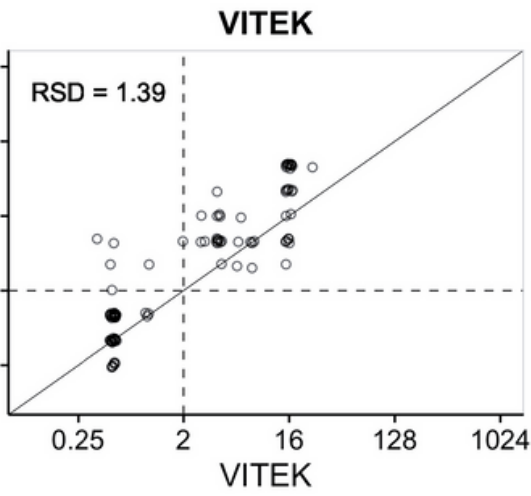

Concentration $(\mu \mathrm{g} / \mathrm{mL})$

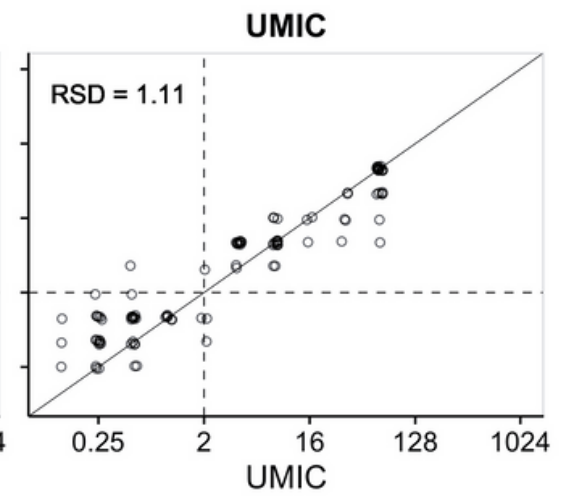

Concentration $(\mu \mathrm{g} / \mathrm{mL})$
Goldstandard vs. VITEK

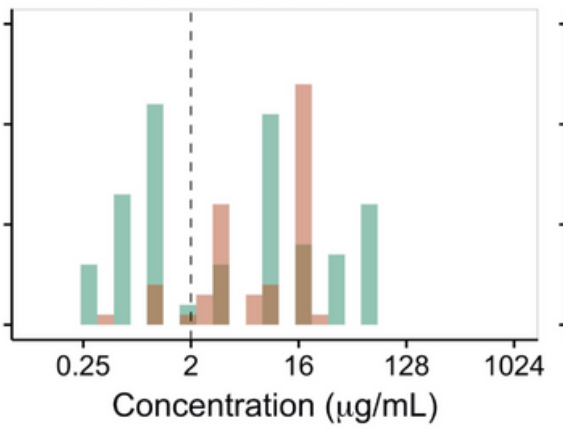

Goldstandard vs. UMIC

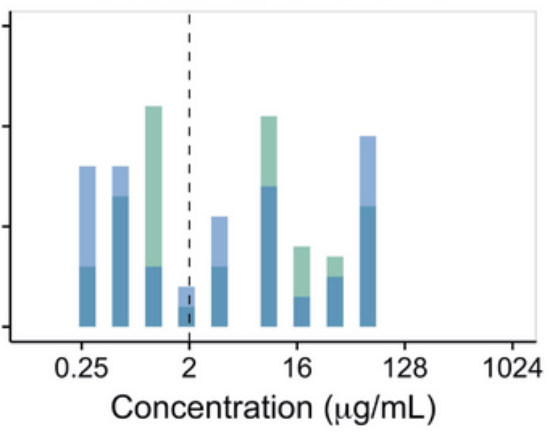




\section{Figure 2}

Distribution of MICs in BDM vs. respective phenotypic test

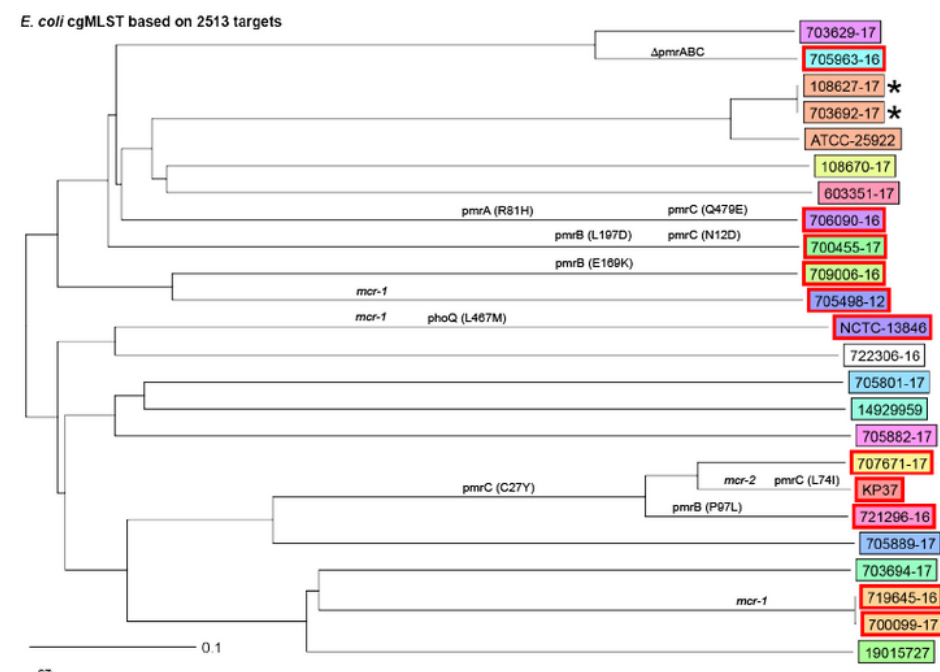

$\mathrm{O}_{167} \mathrm{O}_{10} \mathrm{O}_{205} \mathrm{O}_{38} \mathrm{O}_{409} \mathrm{O}_{504} \mathrm{O}_{744}$ $\begin{array}{lllllll}O_{73} & O_{12} & O_{2673} & O_{404} & O_{428} & O_{537} & O_{95}\end{array}$ O 155 O 131 O 345 O 405 O 457 O 69 Onogroup assigned

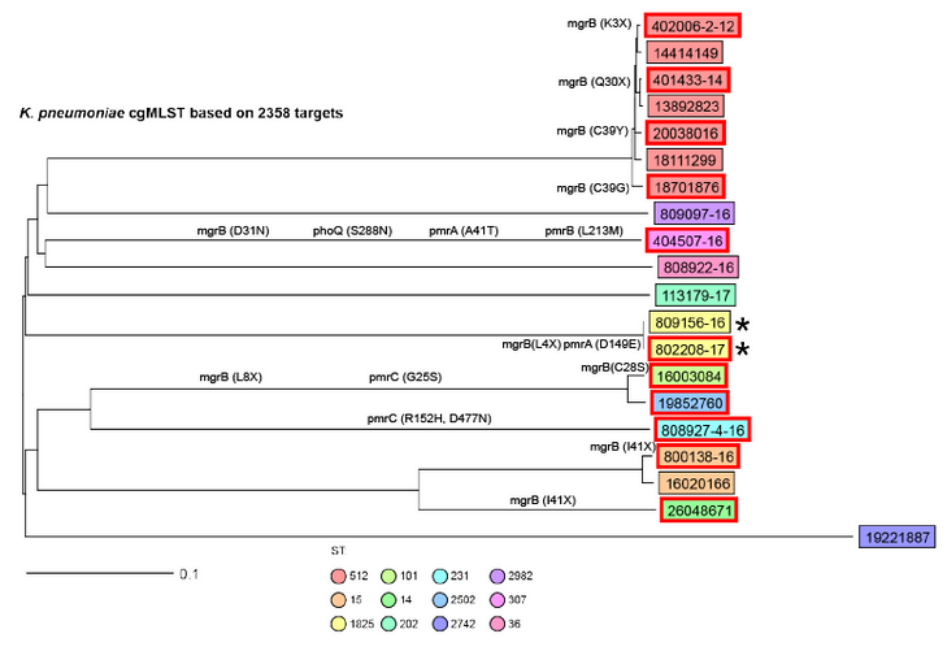

\section{Figure 3}

Core genome MLST Neighbour Joining trees of E. coli and K. pneumoniae. MLST sequence types are shown. Scale bar indicates the number of variant alleles relative to the total number of targets for that species. * indicates isolates from the same patient. Red boxes around isolate names indicate colistin resistance. Mutations associated with colistin resistance are shown on the relevant branches. Acquired plasmid-mediated genes associated with resistance are also shown on the relevant branches in italics.

\section{Supplementary Files}

This is a list of supplementary files associated with this preprint. Click to download.

- Additionalfile1Speciesdistribution.docx

- Additionalfile2Phenotypicassays.docx

- Additionalfile3Rindifferentbacteriatype.xlsx

- Additionalfile4speciesidentification.docx

- Additionalfile5Klebsiellapneumoniaealignments.pdf 
- Additionalfile6E.colialignments.pdf

- Additionalfile7A.bereziniaealignments.pdf 